\title{
Design and baseline characteristics of the Food4Me study: a web-based randomised controlled trial of personalised nutrition in seven European countries
}

\author{
Carlos Celis-Morales $\cdot$ Katherine M. Livingstone $\cdot$ Cyril F. M. Marsaux $\cdot$ Hannah Forster • \\ Clare B. O'Donovan - Clara Woolhead • Anna L. Macready • Rosalind Fallaize - Santiago Navas-Carretero • \\ Rodrigo San-Cristobal - Silvia Kolossa - Kai Hartwig $\cdot$ Lydia Tsirigoti • Christina P. Lambrinou • \\ George Moschonis • Magdalena Godlewska • Agnieszka Surwiłło Keith Grimaldi • Jildau Bouwman • \\ E. J. Daly • Victor Akujobi • Rick O'Riordan · Jettie Hoonhout • Arjan Claassen • Ulrich Hoeller • \\ Thomas E. Gundersen - Siv E. Kaland • John N. S. Matthews • Yannis Manios • Iwona Traczyk • \\ Christian A. Drevon • Eileen R. Gibney • Lorraine Brennan • Marianne C. Walsh • Julie A. Lovegrove • \\ J. Alfredo Martinez • Wim H. M. Saris • Hannelore Daniel • Mike Gibney • John C. Mathers
}

Received: 18 September 2014/ Accepted: 1 December 2014/Published online: 10 December 2014 (C) Springer-Verlag Berlin Heidelberg 2014

\begin{abstract}
Improving lifestyle behaviours has considerable potential for reducing the global burden of non-communicable diseases, promoting better health across the lifecourse and increasing well-being. However, realising this potential will require the development, testing and implementation of much more effective behaviour change
\end{abstract}

On behalf of the Food4Me study.

Trial registration-NCT01530139 (http://clinicaltrials.gov/show/ NCT01530139).

Electronic supplementary material The online version of this article (doi:10.1007/s12263-014-0450-2) contains supplementary material, which is available to authorized users.

C. Celis-Morales · K. M. Livingstone · J. C. Mathers $(\bowtie)$ Human Nutrition Research Centre, Institute of Cellular Medicine, Newcastle University, Campus for Ageing and

Vitality, Newcastle upon Tyne NE4 5PL, UK

e-mail: john.mathers@newcastle.ac.uk

C. F. M. Marsaux

Department of Human Biology, NUTRIM School for Nutrition,

Toxicology and Metabolism, Maastricht University Medical

Centre, Maastricht, The Netherlands

H. Forster · C. B. O'Donovan - C. Woolhead .

E. R. Gibney - L. Brennan - M. C. Walsh .

W. H. M. Saris · M. Gibney

UCD Institute of Food and Health, University College Dublin,

Belfield, Dublin 4, Republic of Ireland

A. L. Macready - R. Fallaize $\cdot$ J. A. Lovegrove

Hugh Sinclair Unit of Human Nutrition and Institute for Cardiovascular and Metabolic Research, University of Reading, Reading, UK interventions than are used conventionally. Therefore, the aim of this study was to conduct a multi-centre, web-based, proof-of-principle study of personalised nutrition (PN) to determine whether providing more personalised dietary advice leads to greater improvements in eating patterns and health outcomes compared to conventional populationbased advice. A total of 5,562 volunteers were screened across seven European countries; the first 1,607 participants who fulfilled the inclusion criteria were recruited into the trial. Participants were randomly assigned to one of the following intervention groups for a 6-month period: Level 0 - control group-receiving conventional, non-PN advice; Level 1-receiving PN advice based on dietary intake data alone; Level 2-receiving PN advice based on dietary

S. Navas-Carretero · R. San-Cristobal · J. Alfredo Martinez Department of Nutrition, Food Science and Physiology, University of Navarra, Pamplona, Spain

S. Navas-Carretero $\cdot$ J. Alfredo Martinez CIBER Fisiopatología Obesidad y Nutrición (CIBERobn), Instituto de Salud Carlos III, Madrid, Spain

S. Kolossa $\cdot$ K. Hartwig $\cdot$ H. Daniel

Biochemistry Unit, ZIEL Research Center of Nutrition and Food

Sciences, Technische Universität München, Munich, Germany

L. Tsirigoti - C. P. Lambrinou - G. Moschonis · Y. Manios Department of Nutrition and Dietetics, Harokopio University, Athens, Greece

M. Godlewska · A. Surwiłło · I. Traczyk

National Food and Nutrition Institute (IZZ), Warsaw, Poland 
intake and phenotypic data; and Level 3-receiving PN advice based on dietary intake, phenotypic and genotypic data. A total of 1,607 participants had a mean age of 39.8 years (ranging from 18 to 79 years). Of these participants, $60.9 \%$ were women and $96.7 \%$ were from white-European background. The mean BMI for all randomised participants was $25.5 \mathrm{~kg} \mathrm{~m}^{-2}$, and $44.8 \%$ of the participants had a BMI $\geq 25.0 \mathrm{~kg} \mathrm{~m}^{-2}$. Food4Me is the first large multi-centre RCT of web-based PN. The main outcomes from the Food4Me study will be submitted for publication during 2015.

Keywords Personalised nutrition - Web-based . Phenotype - Genotype - Randomised controlled trial

\section{Background}

Poor diet, lack of physical activity (PA) and obesity are major risk factors for non-communicable diseases (NCDs) including type 2 diabetes (T2D), cardiovascular disease (CVD) and some cancers (WHO 2009). CVD alone accounts for more than half of the deaths caused by NCDs and is the primary cause of mortality in the European Union, resulting in more than 4 million deaths annually (Nichols et al. 2012). Given that NCDs are predicted to account for $\sim 73 \%$ of global mortality by 2020 (WHO 2008), prevention strategies for NCDs are a priority worldwide. The adoption of a healthy lifestyle, including improved diet and increased PA, is primary prevention strategies to reduce the risk of NCDs (Nichols et al. 2012; WHO 2008), and such changes could prevent up to $80 \%$ of coronary heart disease and cerebrovascular disease (WHO 2008). To date, most strategies to prevent or reduce the incidence of CVD have been targeted at a population level based on "one size fits all" public health recommendations. However, the global burden of NCDs worldwide continues

\section{K. Grimaldi}

Eurogenetica Ltd, 7 Salisbury Road, Burnham-on-Sea, UK

\section{J. Bouwman}

Microbiology and Systems Biology Group, TNO, Utrechtseweg 48, 3704 HE Zeist, The Netherlands

\section{E. J. Daly · V. Akujobi · R. O'Riordan}

Crème Global, Trinity Technology and Enterprise Campus, Grand Canal Quay, Dublin 2, Ireland

\section{J. Hoonhout}

Experiences Research Department, Philips Research, High Tech Campus 34, 5656 AE Eindhoven, The Netherlands

A. Claassen

Software Products and Systems, PGI, Philips Innovation Services, High Tech Campus 33, 5656 AE Eindhoven, The Netherlands to rise (Nichols et al. 2012; WHO 2008, 2009), and more effective prevention strategies are needed.

The concept of personalised nutrition $(\mathrm{PN})$, or nutrition adapted to specific personal characteristics, is not new. Recommendations based on age or physiological status, e.g. infants or pregnant women (FAO 2004), or diseases such as hypertension (Appel et al. 2006) or diabetes (Dyson et al. 2011), have been tailored to meet specific nutritional needs. However, recent progress in the field of genomic research may offer a further level of specificity by providing a rational basis for individual-level, genotype-based nutritional advice, thereby increasing the efficacy of interventions designed to reduce the risk of NCDs (Gibney and Walsh 2013). The idea that genetic information could be used to promote behavioural changes is consistent with psychological theories, such as the protection motivation theory (Rosenstock 1988), that identifies perception of risk as important for driving risk-reducing actions. With this model, a person receiving feedback indicating a higher genetic risk may have greater motivation to change behaviour. However, if "genetic determinism" prevails, people may adopt a fatalistic attitude towards health improvement or maintenance because their genetic profile cannot be modified. However, the opposite effect is also possible, i.e. an individual with a "protective genotype" may adopt an unhealthy lifestyle because they feel they are genetically protected (Senior et al. 1999). A systematic review of the evidence for lifestyle behavioural changes following provision of disease risk predisposition based on DNA information concluded that claims that receiving DNA-based test results motivates people to change their behaviour are not supported by the evidence and that larger and higher quality randomised controlled trials (RCTs) are needed (Marteau et al. 2010). However, a subsequent RCT reported that individuals found dietary recommendations based on genotypic information more understandable and more useful than general dietary advice (Nielsen and El-Sohemy 2012). Whether

U. Hoeller

DSM Nutritional Products, Analytical Research Centre, Basel, Switzerland

T. E. Gundersen · S. E. Kaland

Vitas AS, Gaustadalleen 21, 0349 Oslo, Norway

J. N. S. Matthews

School of Mathematics and Statistics, Newcastle University, Newcastle, UK

C. A. Drevon

Department of Nutrition, Institute of Basic Medical Sciences, Faculty of Medicine, University of Oslo, Oslo, Norway 
this greater understandability was translated into greater dietrelated behaviour change is not known.

Although PN is often considered in the context of dietgene interactions, nutritional advice could also be personalised on other bases including dietary and/or phenotypic information (Gibney and Walsh 2013). Currently, there is a lack of evidence about the relative effectiveness of PN advice based on dietary, phenotypic and genotypic information. Therefore, the aim of the Food4Me RCT was to conduct a multi-centre, proof-of-principle (PoP) study of PN delivered at three intervention levels to determine whether providing more personalised dietary advice leads to greater dietary improvements and health outcomes compared with conventional population-based advice. In this paper, we report the study design and protocol for the Food4Me PoP study as well as the baseline characteristics of the participants recruited into the study.

\section{Methods}

Study design

The Food4Me PoP study was a four-arm, web-based RCT conducted across seven European countries, which compared the effects of different levels of PN on health-related outcomes. The intervention was designed to emulate a reallife web-based PN service, and the study aimed to answer the following primary research questions:

- Does personalisation of dietary advice assist and/or motivate participants to eat a healthier diet in comparison with non-personalised, conventional healthy eating guidelines?

- Is personalisation based on individualised phenotypic or genotypic information more effective in assisting and/or motivating study participants to make, and to sustain, appropriate healthy changes, than personalisation based on diet alone?

To answer these research questions, we used an hierarchical study design in participants randomised to a control group (Level 0) or to one of $3 \mathrm{PN}$ interventions with increasingly complex bases for personalised dietary advice (Levels 1-3), i.e. randomisation was to one of the following treatment groups for a 6-month period:

- Level 0 (LO): (control group): non-personalised dietary advice based on (European) population healthy eating guidelines.

- Level 1 (L1): personalised dietary advice based on individual dietary intake data alone.

- Level 2 (L2): personalised dietary advice based on individual dietary intake and phenotypic data.
- Level 3 (L3): personalised dietary advice based on individual dietary intake, phenotypic and genotypic data.

The secondary research question of the study was as follows:

- Does more frequent feedback help participants to improve their compliance and motivate them to eat a healthier diet and follow a healthier lifestyle in comparison with those receiving less frequent feedback?

To answer this secondary research question, participants randomised to Levels 1,2 or 3 were further randomised into "low-intensity" or "high-intensity" intervention groups:

- Low intensity: personalised feedback given three times during the intervention (at baseline, month 3 and month 6).

- High intensity: personalised feedback given five times during the intervention (at baseline and months 1, 2, 3 and 6). In addition, the "high-intensity" group had access to an online forum for discussion of topics related to the intervention, had access to personalised recipes and had more personalised physical activity (PA) feedback.

Primary and secondary outcomes

The primary outcome of the study was dietary intake at months 3 and 6. The secondary outcomes included PA and phenotypic biomarkers at months 3 and 6 . The latter included obesity-related measures (i.e. body weight, body mass index (BMI) and waist circumference) and bloodbased biomarkers (i.e. blood glucose, total cholesterol, carotenoids and fatty acids).

\section{Recruitment}

Participants were recruited via the Internet to emulate a web-based PN service. This was aided by local and national advertising of the study via the Internet, radio, newspapers, posters, e-flyers, social media and word of mouth.

Recruitment into the Food4Me intervention trial was carried out using identical standardised protocols in seven European recruitment centres. Based on sample size calculations (see below for further details), we aimed to recruit a total of 1,540 study participants (i.e. 220 participants per country). The PoP study recruitment sites were as follows: University College Dublin, Ireland; Maastricht University, the Netherlands; University of Navarra, Spain; Harokopio University, Greece; University of Reading, UK; 
National Food and Nutrition Institute, Poland; and Technische Universität München, Germany.

\section{Eligibility criteria}

Participants aged $\geq 18$ years of age were included in the study. To keep the cohort as representative as possible of the adult population, the following minimal sets of exclusion criteria were applied:

- Pregnant or lactating;

- No or limited access to the Internet;

- Following a prescribed diet for any reason, including weight loss, in the last 3 months;

- Diabetes, coeliac disease, Crohn's disease, or any metabolic disease or condition altering nutritional requirements such as thyroid disorders (if condition was not controlled), allergies or food intolerances.

Exclusion based on prescribed diet or specific diseases was to avoid the theoretical risk that participating in the study could be disadvantageous to the individual.

Ethical approval and participant consent

The Research Ethics Committees at each University or Research Centre delivering the intervention granted ethical approval for the study. An application for the Norwegian arm of the study administered by the University of Oslo was not approved by the local ethics committee (details will be reported elsewhere).

Prior to participation, an information sheet was provided to all potential volunteers who completed an online informed consent form before submitting personal data. This signed online consent form was automatically directed to the study coordinator to be counter-signed and archived. A second online informed consent form was completed before randomisation to the intervention study only for participants who met the inclusion criteria. A two-step consenting process was applied to permit collection of socio-demographic and dietary information for those interested in participating in PN even if they were ineligible for enrolment in this study, e.g. because of prescribed diets or food allergies. All Ethical Committees accepted an online informed consent procedure, except for the Netherlands and Germany whose ethics committees requested an additional written informed consent form for each participant recruited into the study. This hard copy consent form was returned by the participant to the respective recruitment centre.

Intervention design

Eligible and consenting participants were allocated to one of the four arms of the study, which included three intervention groups receiving different levels of personalised nutritional advice (L1: dietary data only; L2: dietary and phenotypic data; and L3: dietary, phenotypic and genotypic data) and the control group (L0), receiving conventional, non-personalised advice. To address our secondary research question, participants in levels L1, L2 and L3 were allocated into "low-" or "high-"intensity groups (see next section for details of the randomisation methods). Participants were asked to complete the data and sample collection summarised in Table 1 . At the end of the study (month 6), all participants received a personalised report which contained dietary, phenotypic and genotypic information and which summarised changes in their individual dietary intake and phenotypic measures between baseline and month 6 of the intervention.

\section{Randomisation}

Participants were randomised to one of the seven treatment groups (control group (L0), L1 high intensity, L1 low intensity, L2 high intensity, L2 low intensity, L3 high intensity and L3 low intensity) in combination with stratified randomisation by country (UK, Greece, Spain, Poland, Ireland, Germany and the Netherlands), sex (female or male) and age ( $<45$ or $\geq 45$ years) equally allocated to each treatment using an urn randomisation scheme (Wei and Lachin 1988).

Intervention groups

Level 0 ("control group")

Following baseline measures, participants randomised to the control group (L0) received non-personalised dietary advice based on conventional population healthy eating guidelines. This non-personalised dietary advice was based on national dietary recommendations in each of the seven European countries participating in the Food4Me PoP Study which were integrated to produce a coherent set of recommendations suitable for Europe-wide use. These "standardised" recommendations included advice on energy intake to optimise BMI and on the consumption of fruits and vegetables, whole-grain products, fish, dairy products, meat, type of fat and salt. In addition, these recommendations included a generic $\mathrm{PA}$ recommendation (Table 2). An advice leaflet was delivered via the web and also attached to an e-mail, which was sent to participants at baseline and at month 3 of the study (Appendix II in Supplementary Material).

Level 1 ("diet group")

Following baseline measures, participants randomised to L1 received feedback on how their intakes of specific food 
Table 1 Summary of data and biological samples collected at each time point during the intervention a Only weight and height were collected at second screening questionnaire

b Measures taken at month 1 and 2 were only for the highintensity group

\begin{tabular}{|c|c|c|c|c|c|c|c|}
\hline \multirow[t]{2}{*}{ Outcomes } & \multicolumn{7}{|c|}{ Time point } \\
\hline & $\begin{array}{l}\text { First } \\
\text { screening }\end{array}$ & $\begin{array}{l}\text { Second } \\
\text { screening }\end{array}$ & $\begin{array}{l}\text { Month } 0 \\
\text { (baseline) }\end{array}$ & $\begin{array}{l}\text { Month } \\
1^{\mathrm{b}}\end{array}$ & $\begin{array}{l}\text { Month } \\
2^{\mathrm{b}}\end{array}$ & $\begin{array}{l}\text { Month } \\
3\end{array}$ & $\begin{array}{l}\text { Month } \\
6\end{array}$ \\
\hline $\begin{array}{l}\text { Socio-demographic data (name, } \\
\text { age, gender) }\end{array}$ & $\checkmark$ & $\checkmark$ & & & & & \\
\hline $\begin{array}{l}\text { Eligibility criteria (pregnancy, } \\
\text { therapeutic diet, food allergy or } \\
\text { intolerance, internet access) }\end{array}$ & $\checkmark$ & $\checkmark$ & & & & & \\
\hline First online consent & $\checkmark$ & & & & & & \\
\hline $\begin{array}{l}\text { Second socio-demographic data } \\
\text { (age, gender, address, ethnicity) }\end{array}$ & & $\checkmark$ & & & & & \\
\hline $\begin{array}{l}\text { Health-related questionnaire } \\
\text { (weight, height, medical health } \\
\text { status, smoking, sun exposure) }\end{array}$ & & $\boldsymbol{\alpha}$ & & & & & \\
\hline $\begin{array}{l}\text { Food choice and eating habits } \\
\text { questionnaire }\end{array}$ & & $\checkmark$ & & & & & \\
\hline Health perception questionnaire & & $\checkmark$ & & & & & \\
\hline Second online consent & & $\checkmark$ & & & & & \\
\hline $\begin{array}{l}\text { Online Food Frequency } \\
\text { Questionnaire (FFQ) }\end{array}$ & & $\checkmark$ & $\checkmark$ & $\checkmark$ & $\checkmark$ & $\checkmark$ & $\checkmark$ \\
\hline $\begin{array}{l}\text { Anthropometrics measures } \\
\text { (weight, height, waist, hip and } \\
\text { upper leg circumference) }\end{array}$ & & $\boldsymbol{\nu}^{\mathrm{a}}$ & $\checkmark$ & $\checkmark$ & $\checkmark$ & $\checkmark$ & $\checkmark$ \\
\hline $\begin{array}{l}\text { Buccal cell collection for genetic } \\
\text { analysis }\end{array}$ & & & $\checkmark$ & & & & \\
\hline Dry blood spot collection & & & $\checkmark$ & & & $\checkmark$ & $\checkmark$ \\
\hline $\begin{array}{l}\text { Baecke Physical Activity } \\
\text { Questionnaire }\end{array}$ & & & $\checkmark$ & $\checkmark$ & $\checkmark$ & $\checkmark$ & $\checkmark$ \\
\hline $\begin{array}{l}\text { Physical activity levels } \\
\text { (accelerometer) }\end{array}$ & & & $\checkmark$ & $\checkmark$ & $\checkmark$ & $\checkmark$ & $\checkmark$ \\
\hline
\end{tabular}

groups (fruits and vegetables, whole-grain products, fish, dairy products and meat) compared with guideline amounts. In addition, personalised dietary advice based on their reported dietary intake at baseline and month 3 (Table 2).

\section{Level 2 ("diet + phenotype group")}

Following baseline measures, participants randomised to L2 received personalised dietary advice based on their dietary intake (as for L1) and also on their baseline phenotypic data. The phenotypic feedback was based on anthropometric measurements and nutrient- and metabolicrelated biomarkers (Table 2).

\section{Level 3 ("diet + phenotype + genotype group")}

Participants randomised to L3 received personalised dietary advice based on their dietary intake plus phenotypic and genotypic data collected at baseline. The genotypic feedback was based on specific variants in five nutrient- responsive genes selected specifically for the study. A description of these five genes and the related dietary factors is given in supplementary material (Table S2).

Personalised feedback report

Participants randomised to L1, L2 and L3 received personalised feedback based on decision trees developed to provide a structured, evidence-based protocol for delivering tailored advice. This advice was based on dietary, PA, phenotypic and genotypic information as appropriate for each intervention group. In each case, intakes were compared with recommended intakes and determined to be adequate, high or low. If intakes were categorised as too high or too low, contributing foods were identified and specific messages were developed to advise change in intake of those foods. Full details of these decision trees will be published elsewhere. Protocols for the decision trees were standardised across the seven recruitment centres and translated into the language of each country. Nutritionists and dietitians implementing the decision trees 


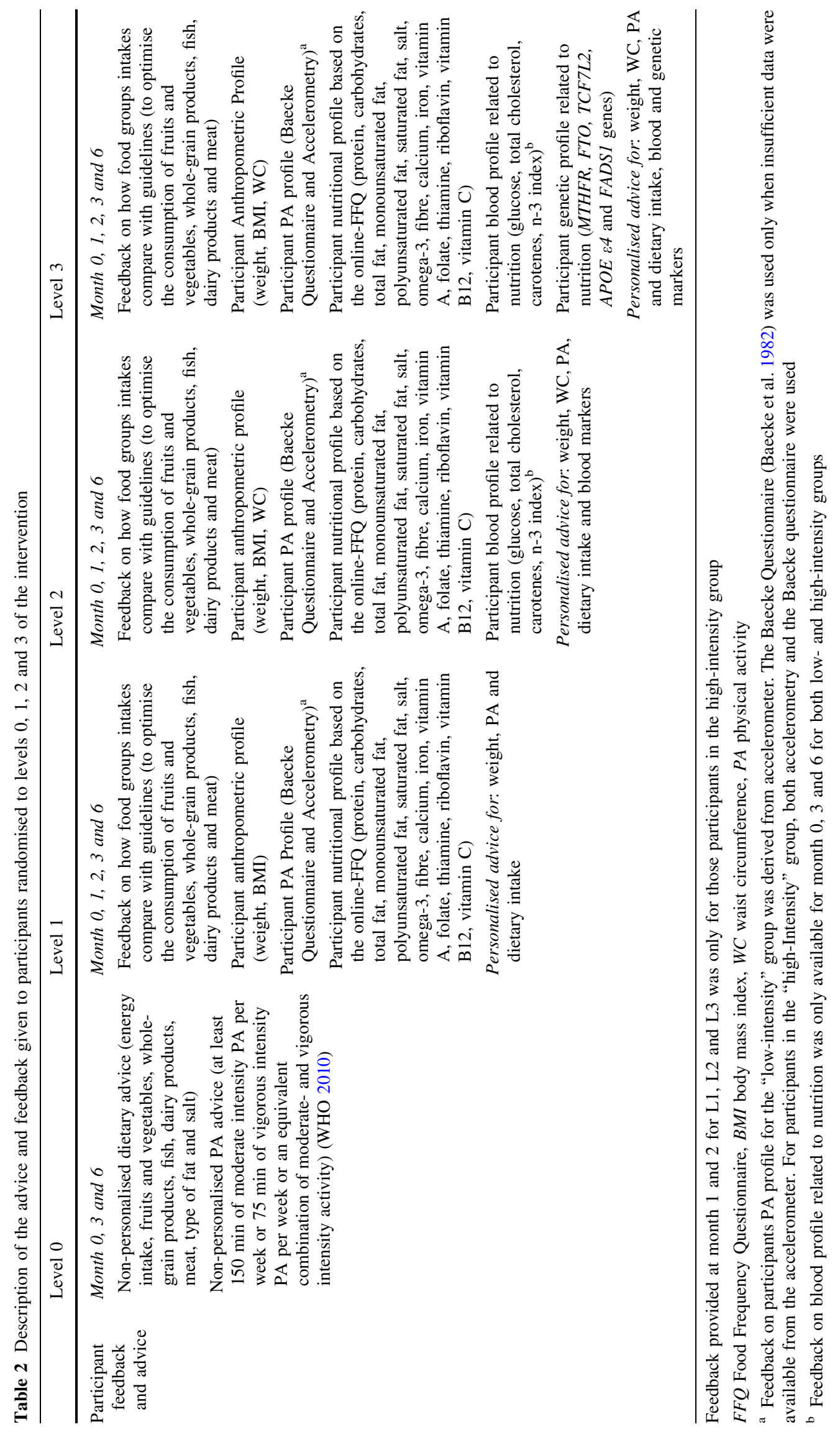


were trained to ensure consistency in the PN advice given throughout the study, and, across all seven countries, these staff participated in frequent teleconferences (every 1-2 weeks) to resolve issues and to share best practice.

The participants' reports contained information on how their health-related characteristics compared with recommendations. Estimations of healthy behaviours were explained using a three-colour sliding scale: green representing "Good, no change recommended", amber representing "Improvement recommended" and red representing "Improvement strongly recommended". For the genotype-based information, risk was indicated using "Yes" or "No" according to whether the participant did, or did not, carry the higher risk variant for each of the five nutrient-related genes as specified in Table 2. Finally, each report contained a personalised message from the dietitian/ nutritionist to the participant. This message provided tailored advice for body weight and PA, and included specific nutrition-related goals derived from dietary, phenotypic and/or genotypic markers (according to the participants' intervention group). Based on patient-centred counselling models for facilitating dietary change (Rosal et al. 2001), a total of three nutrient-related goals were provided. These goals were selected by ranking all dietary, phenotypic and genotypic markers (as appropriate for the intervention group) based on their risk status (red, amber or green). The cut-off points for each of the nutritional and phenotypic variables were used to derive personalised goals and advice (Appendix II and II in Supplementary material).

\section{Behavioural change techniques}

Explicit behaviour change techniques (BCT) were integrated into several aspects of the intervention and used to support, encourage and enhance dietary and lifestyle changes. The BCT and their conceptual framework were derived from work by Michie et al. on smoking cessation and dietary behaviour change (Michie et al. 2011a, b). The BCT categories used in the Food4Me PoP study were as follows: (1) behaviour and motivation, (2) behaviour and self-regulatory capacity/skills, (3) interaction and delivery, (4) interaction and information gathering and (5) interaction and communication. More details about the BCTs used in this study will be reported separately.

\section{Study measures}

Participants consented to self-report all their measures via the Internet and to send requested biological samples (Dry Blood Spot cards and buccal swabs) by conventional mail, using prepaid, stamped addressed envelopes provided by the research team. To ensure that procedures were similar in all recruiting centres, standardised operating procedures were prepared for all study procedures (see below), and researchers underwent centralised training. In addition, to enable participants to collect and report the required information and to collect, process and dispatch the necessary biological samples correctly, participants were provided with detailed instructions online, including pictures and video demonstrations of all procedures, in their native language. Time points for each measurement are summarised in Table 1.

\section{First screening questionnaire}

Participants who consented to take part in the study completed an online screening questionnaire that included basic socio-demographic and health statistics, and information about Internet access, pregnancy and lactation, prescribed diets, food intolerance and allergies (used as exclusion criteria). Persons who were deemed unsuitable for the study, e.g. because of inadequate Internet access, pregnancy or use of a therapeutic diet, received formal e-mail notification that they did not match the inclusion criteria for the study and were thanked for their time.

\section{Second screening questionnaire}

Eligible participants for inclusion in the RCT completed a second online questionnaire, which collected more detailed socio-demographic, health and anthropometric data, as well as detailed information on food choices and dietary habits using a Food Frequency Questionnaire (FFQ) developed and validated specifically for this study (see below). Following assessment of this information, participants considered suitable for inclusion in the intervention study were asked to complete a second online consent form, which was sent to the study coordinator to be signed and archived. Potential participants considered unsuitable for the intervention study, e.g. through non-compliance in completion of the screening FFQ, received formal notification that they did not match the inclusion criteria for the study and were thanked for their time.

\section{Anthropometric measurements}

Body weight, height and upper thigh, waist and hip circumferences were self-measured and self-reported by participants via the Internet. Standardised instructions on how to perform these measurements were provided in printed and digital format (i.e. a video clip available on the Food4Me website in the languages of each of the seven recruitment countries). Participants were instructed to measure body weight without shoes and wear light clothing using a home or commercial scale and to measure height barefoot using a standardised measuring tape provided by 
Food4Me. Waist circumference was measured at the midpoint between the lower rib and the iliac crest using the same tape measure. Hip circumference was measured at the widest point around the greater trochanters, while the upper thigh circumference was measured midway between the iliac crest and the knee.

\section{Food Frequency Questionnaire (FFQ)}

Habitual dietary intake was quantified using an onlineFFQ, developed for this study which included food items consumed frequently in each of the seven recruitment countries. The Food4Me online-FFQ has been validated against a 4-day weighed food record, and the agreement between methods varied, with correlations ranging from .23 (vitamin D) to .65 (protein, \% total energy) for nutrient intakes and .11 (soups, sauces and miscellaneous foods) to .73 (yogurts) for food group intake (Fallaize et al. 2014; Forster et al. 2014). Intakes of foods and nutrients were computed in real time using a food composition database based on McCance \& Widdowson's "The composition of foods" (McCance 2002).

\section{Metabolic markers}

Finger-prick blood samples were collected by participants using a collection pack provided by Vitas Ltd, Oslo, Norway. To help with blood collection, participants had access to an online video demonstration with instructions and frequently asked questions. Each participant was asked to fill two Dry Blood Spot cards (equivalent to five drops of blood or to $150 \mu \mathrm{l}$ of blood per card) at each collection time point. When the ten blood spots were filled, participants were instructed to dry the cards at room temperature for at least $2 \mathrm{~h}$, but not longer than $4 \mathrm{~h}$, before samples were put in an airtight aluminium bag with drying sachet and returned by post to the corresponding recruiting centre. The centres shipped the samples to Vitas (Vitas Ltd, Norway) and DSM (DSM Nutritional Products Ltd, Switzerland) for measurements of glucose, total cholesterol, carotenoids, n-3 fatty acid index and 32 other fatty acids (by Vitas), and vitamin D (25-OH D2 and 25-OH D3) (by DSM) (Table S1 in Supplementary Material).

\section{Genotypic analyses}

Buccal cell samples were collected by participants at baseline using Isohelix SK-1 DNA buccal swabs and Isohelix Dri-capsules and returned by post to each recruiting centre for shipment to LCG Genomics (Hertfordshire, UK). LCG Genomics undertook DNA extraction and genotyping of the five loci used for derived personalised advice (Table
S2 in Supplementary Material). These loci were analysed using $\mathrm{KASP}^{\mathrm{TM}}$ genotyping assays to provide bi-allelic scoring of single nucleotide polymorphisms (SNPs) and insertions and deletions at specific loci.

\section{Physical activity}

PA patterns were determined using a PA monitor-the DirectLife triaxial accelerometer for movement registration (TracmorD) (Philips Consumer Lifestyle, the Netherlands; Bonomi et al. 2010; Plasqui et al. 2005; Plasqui and Westerterp 2007)—and a self-reported Baecke PA questionnaire (Baecke et al. 1982) which was completed online. The accelerometer-based monitor (Philips DirectLife Activity Monitor, the Netherlands) was posted to each participant. Online video demonstrations as well as digital and printed instructions were provided at baseline. Participants were instructed to wear the monitor throughout the six-month intervention and to upload their PA data fortnightly via an online interface.

\section{Sample size consideration}

A power calculation was conducted a priori using Mini$\operatorname{tab}^{\circledR}$ (version 16.1.0) and data for n-3 fatty acids and glucose concentrations in adult European populations. To address our primary research questions, and based on the resources available for the intervention, a sample size of $n=326$ participants for each of the four intervention arms was planned. This allows us to detect differences of 0.22 $\mathrm{SD}$ in our main outcomes with $80 \%$ power and alpha $=0.05$. Assuming that the population standard deviation (SD) for $\mathrm{n}-3$ fatty acid index is 1.5 units and for glucose is $1.05 \mathrm{mmol} \mathrm{l}^{-1}$, a total sample of $n=1,280$ participants was estimated as sufficient to detect a real differences of 0.33 units for $\mathrm{n}-3$ PUFA and $0.23 \mathrm{mmol} \mathrm{l}^{-1}$ glucose post-intervention. Allowing for a potential $20 \%$ drop out, we aimed to recruit 1,540 participants into the study (220 participants per centre).

\section{Statistical analysis}

Data will be analysed on an intention-to-treat basis. Responses to the intervention for primary (dietary) and secondary (non-dietary: anthropometrics, PA and bloodbased biomarkers) outcomes will be analysed using two approaches.

To answer our primary research question ("does personalisation of dietary advice produce larger and more appropriate changes in dietary intake?"), intakes by those randomised to control (L0) will be compared with intakes of those randomised to L1-L3. For this, treatment groups (control versus L1-L3) will be compared using analysis of 
covariance, with pre-defined orthogonal (single degree of freedom) contrasts to test our a priori hypotheses. Contrast 1: comparison of L0 with the mean of L1, L2 and L3 provides a test of the overall effect of PN versus the control (non-personalised dietary advice). Contrast 2: comparison of L1 with the mean L2 and L3 tests whether personalisation based on phenotypic and or genotypic information differs from that based on dietary assessment only. Finally, Contrast 3 comparison or L2 with L3 tests whether the addition of genotypic information produces effects which differ from that using phenotypic information only. Generic dietary targets set for LO (energy intake, fruit and vegetables, whole grain, dairy products, oily fish, red meat, salt, fats and physical activity) will be used as outcome measures for each dietary target separately. To answer our secondary research question ("Does more frequent feedback produce bigger beneficial changes?"), further analysis will be undertaken using a $2 \times 3$ factorial analysis including PN treatments (L1, L2, and L3) and intensity groups (high and low) for each of the outcomes. The same approach will be applied to the non-dietary outcomes (weight, BMI, waist circumference, PA and blood-derived biomarkers including glucose, total cholesterol, carotenoids and n-3 fatty acid index).

Our second approach will recognise that each participant randomised to $\mathrm{L} 1, \mathrm{~L} 2$ or L3 will receive individualised tailored dietary targets. Thus, outcomes for each tailorednutrient advice target will be compared between L1-L3 groups and participants in L0 who were not given personalised advice. The same approach will be applied to the investigation of the effects of the intervention on the nondietary outcomes (anthropometrics, PA and blood-based biomarkers including glucose, total cholesterol, carotenoids and n-3 fatty acids).

\section{Results}

A total of 5,562 participants $(63.2 \%$ females $)$ were screened online over a 12-months period between August 2012 and August 2013. Of these, the first 1,607 (28.9\%) volunteers meeting the inclusion criteria were recruited into the RCT and randomised to one of the four intervention groups of the study (Fig. 1). Baseline characteristics of the participants are shown in Table 3. Participants aged 18-79 years (mean 39.8, SD 13.1) were included in the study of whom $60.9 \%(n=979)$ were women and $96.8 \%$ ( $n=1,556)$ were from a white-European ethnic background. The mean BMI for all randomised participants was 25.5 (SD 5.2) $\mathrm{kg} \mathrm{m}^{-2}$, and $44.8 \%(n=721)$ of participants were overweight or obese (BMI $\left.\geq 25.0 \mathrm{~kg} \mathrm{~m}^{-2}\right)$. As a measure of central adiposity, $24.5 \%(n=394)$ of participants had a waist circumference higher than $88 \mathrm{~cm}$ and
$102 \mathrm{~cm}$ for women and men, respectively. Based on selfreported PA using the online Baecke PA questionnaire, 42.8 and $18.6 \%$ of the cohort were physically active during leisure time and work, respectively. In addition, $12.1 \%(n=195)$ of the participants were current smokers.

\section{Discussion}

In this study, we describe the rational, design and baseline characteristic of participants who took part in a panEuropean PN study which is aiming to test the hypothesis that a personalised approach to lifestyle-based interventions focusing on nutrition would promote greater, and more appropriate, changes in diet and on health-related outcomes than a conventional, non-personalised approach.

We delivered the intervention via the Internet across seven European countries. Higher proportions of those interested in taking part in the Food4Me study [63.0\% ( $n=3,503$ ) of the screened volunteers] and of the randomised participants [60.9\% $(n=979)]$ were female. These results confirm findings from previous web-based nutritional interventions (Kodama et al. 2012), and from conventional dietary intervention studies (French et al. 1994; Hearty et al. 2007; Sabinsky et al. 2007), showing that females are more likely to volunteer for nutritionrelated research studies. Our study recruited participants from every decade of adult life, from teens to seventies: $1.7 \%$ of randomised participants were aged $<20$ years, $28.3 \%$ aged 20-29 years, 22.4\% aged 30-39 years, $21.4 \%$ aged $40-49$ years, $18.8 \%$ aged 50-59 years, $6.7 \%$ aged 60-69 years and $0.6 \%$ aged $>70$ years. The webbased design of the study is likely to have contributed to the observation that there were fewer participants in the older age groups. Possible explanations for this may include (1) less access to computers and the Internet, although such access is changing fast all over Europe (Seybert 2010); (2) less interest in the concept of PN and genetic testing (Fallaize et al. 2013; Stewart-Knox et al. 2009); (3) less interest in health-related behavioural research generally; and (4) less well targeted by the recruitment advertising. In line with prevalence across Europe, given just under half $(44.8 \%)$ of participants had a BMI $\geq 25.0 \mathrm{~kg} \mathrm{~m}^{-2}$ and $32.1 \%$ were physically inactive individuals. Our results suggest that those interested in PN are representative of European adults (Hallal et al. 2012; OECD 2012) and are not unduly skewed towards individuals who are already very healthy people (the "worried well") or those wishing to lose weight (Fallaize et al. 2013; Gibney and Walsh 2013; Stewart-Knox et al. 2009, 2013). The profile of the European population interested in webdelivered $\mathrm{PN}$ and recruited to the Food4Me intervention study is broadly similar to that of the adult population in 


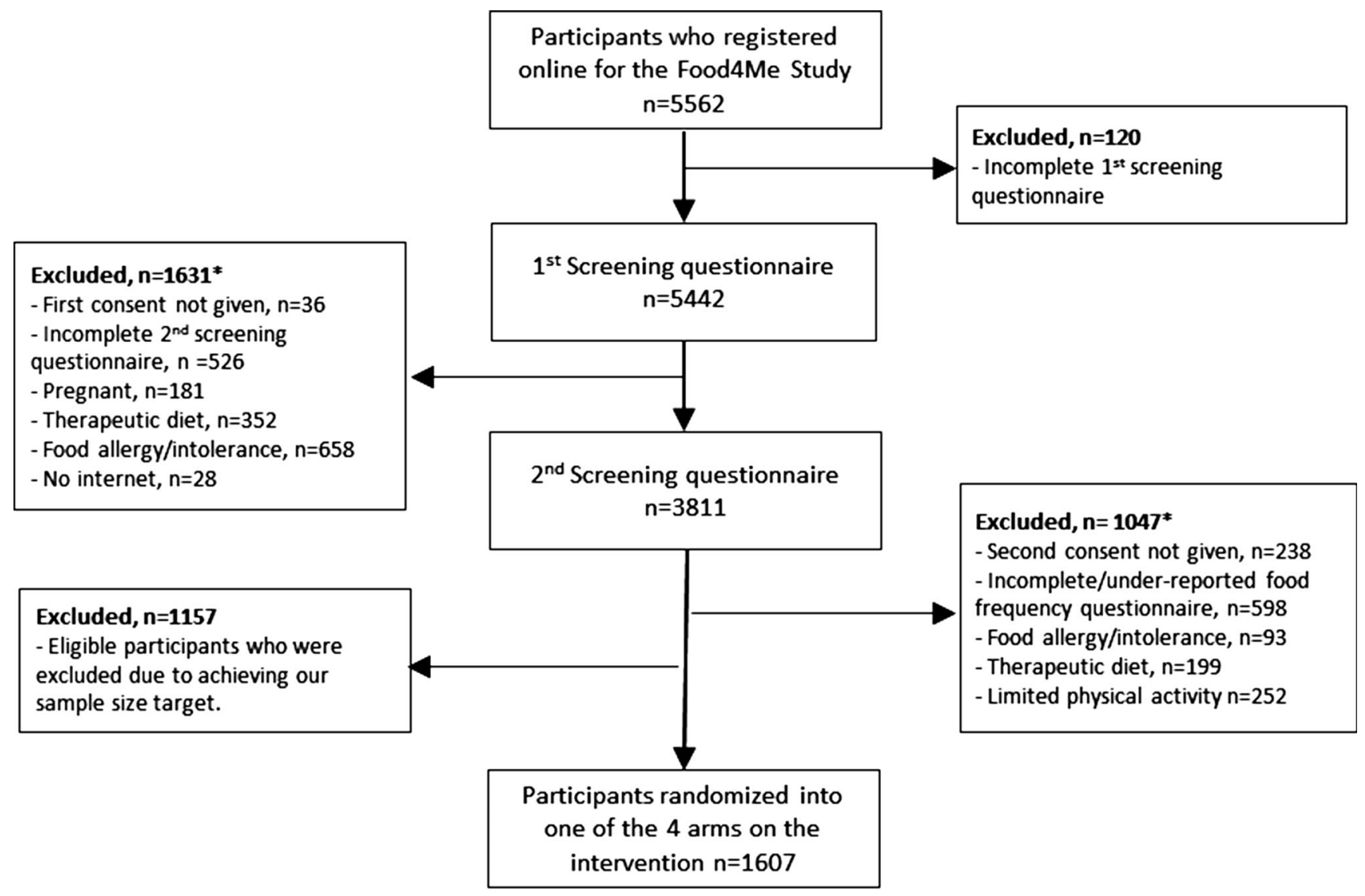

Fig. 1 Food4Me proof-of-principle study CONSORT flowchart. Note: *Total numbers of participants reporting one or more exclusion criteria

Europe most of whom would benefit from improving diet and other lifestyle behaviours.

Strengths and limitations

The Food4Me study is the largest web-based, PN intervention study to date and is expected to provide robust and novel evidence for evaluating the impact of PN on dietary intake and on health-related outcomes. This study is innovative in that it tests the utility of personalisation based on dietary, phenotypic and genotypic information. In addition, the delivery of the intervention was facilitated by an online interface through which participants could interact with the dietitians, nutritionists and researchers at each centre during the 6-month intervention. Other innovative aspects of the Food4Me study include the creation of algorithms for deriving and delivering tailored lifestyle advice on multiple participant characteristics including behavioural (diet and PA), phenotypic and genotypic information (these algorithms will be published elsewhere). A second strength was the delivery of the intervention across seven European countries simultaneously in real time via the web. The Food4Me research team also designed, developed and implemented a remote system for data and biological sample collection enabling participants to provide dietary, anthropometric, PA and other healthrelated information via the Internet, as well as biological samples (dry blood spots and buccal cells) for nutritional, metabolic and genotypic measurements.

Compared with what would be possible in conventional face-to-face interventions, the web-based design of our present study limited the number of measures that were collected. As a result, although participants were well characterised and phenotyped, some key health biomarkers, such as blood pressure, were not measured. Furthermore, all data collected during the study were self-reported or derived from biological samples collected remotely with the potential for introduction of measurement errors. However, previous validation studies of web-based interventions have found good agreement between self-reported and measured height and weight (Bonn et al. 2013; Lassale et al. 2013; Pursey et al. 2014). To minimise such measurement errors, all protocols were standardised across all centres and delivered in their native language. Moreover, participants were assisted in their recording of information and their sample collection by the provision of detailed 
Table 3 Baseline characteristic of the participants randomised to the Food4Me proof-of-principle study

\begin{tabular}{|c|c|}
\hline Demographic & Mean (SD) $(\%)$ \\
\hline Total $(n)$ & 1,607 \\
\hline Sex-female $(\%)$ & 60.9 \\
\hline Age (years) & $39.8(13.1)$ \\
\hline Age range (years) & $18-79$ \\
\hline \multicolumn{2}{|l|}{ Ethnicity (\%) } \\
\hline White $(\%)$ & 96.8 \\
\hline Asia-Chinese $(\%)$ & 0.7 \\
\hline Blacks (\%) & 0.1 \\
\hline Mixed (\%) & 1.4 \\
\hline Other ethnic groups (\%) & 1.1 \\
\hline \multicolumn{2}{|l|}{ Anthropometrics } \\
\hline Height (cm) & $171.1(9.4)$ \\
\hline Weight (kg) & $74.6(15.8)$ \\
\hline BMI $\left(\mathrm{kg} \mathrm{m}^{-2}\right)$ & $25.5(5.2)$ \\
\hline Waist circumference $(\mathrm{cm})$ & $85.6(13.9)$ \\
\hline Hip circumference (cm) & $95.4(12.5)$ \\
\hline \multicolumn{2}{|l|}{ Weight status $(\%)^{\mathrm{a}}$} \\
\hline Underweight & 2.7 \\
\hline Normal weight & 51.1 \\
\hline Overweight & 30.2 \\
\hline Obese & 14.6 \\
\hline Central obesity ${ }^{\mathrm{b}}$ & 24.5 \\
\hline \multicolumn{2}{|l|}{ Other lifestyle factors (\%) } \\
\hline Current smokers & 12.1 \\
\hline Ex-smokers & 25.4 \\
\hline Non-smokers & 62.5 \\
\hline \multicolumn{2}{|l|}{ Physical activity at work ${ }^{\mathrm{c}}$} \\
\hline Sedentary & 23.0 \\
\hline Light to moderately active & 58.4 \\
\hline Active & 18.6 \\
\hline \multicolumn{2}{|c|}{ Physical activity during leisure time ${ }^{c}$} \\
\hline Sedentary & 8.7 \\
\hline Light to moderately active & 48.5 \\
\hline Active & 42.8 \\
\hline
\end{tabular}

Data presented as mean (SD) for continuous variables and as a percentage $(\%)$ for categorical variables

${ }^{\text {a }}$ BMI cut-off points used for weight status classification were as follows: underweight $<18.5 \mathrm{~kg} \mathrm{~m}^{-2}$, normal weight $\geq 18.5$ to $\leq 24.9 \mathrm{~kg} \mathrm{~m}^{-2}$, overweight $\geq 25$ to $\leq 29.9 \mathrm{~kg} \mathrm{~m}^{-2}$, obese $\geq 30.0 \mathrm{~kg} \mathrm{~m}^{-2}$

b Central obesity was defined using waist circumference as a proxy, with sex-specific cut-off points ( $\geq 88 \mathrm{~cm}$ for women and $\geq 102 \mathrm{~cm}$ for men)

${ }^{c}$ Physical activity at work and during sport and leisure time was determined using the Baecke Questionnaire, and individuals were classified using the following cut-off points (sedentary $<1.8$, light to moderately active $1.8-2.8$, active $>2.8$ ) instructions, video clips and frequently asked questions. Remote recruitment and data collection could have introduced problems of identity in the present study, i.e. participants pretending to be someone other than themselves. To address these challenges, $10 \%$ of the randomised participants at each recruitment centre participated in a validation study in which participants visited the centre. During this visit, anthropometric measurements were replicated by a trained researcher to compare to the information provided online, a further buccal cell sample was collected to repeat the genotypic characterisation and the researcher confirmed the participant's age verbally and sex visually. This information will validate the reliability of self-reported data collected via the Internet (results of the validation study will be presented elsewhere).

Implications

Outcomes from the Food4Me PoP Study will provide strong evidence for the effectiveness, or otherwise, of a personalised approach compared with the conventional "one size fits all" generic approach to dietary and lifestyle changes to improve public health. Specifically, this study will demonstrate whether personalisation of dietary advice assists and/or motivates individuals to eat a healthier diet and to follow a healthier lifestyle in comparison with nonpersonalised, conventional dietary advice. Importantly, our trial will elucidate whether provision of individualised feedback and advice based on detailed analysis of habitual diet, phenotypic or genotypic information is more effective in assisting and/or motivating the general population to make, and to sustain, appropriate healthy changes to their habitual diet and lifestyle. Furthermore, the study aims to determine whether more feedback improves participant compliance and results in larger, more appropriate changes in diet and lifestyle in comparison with the provision of less intensive feedback. The pragmatic design of the study, which attempted to emulate key features of a real-life Internet-based PN service, will illustrate the utility of using this medium for the delivery of health-promoting interventions and will establish protocols that can collect information necessary for evidence-based PN advice and support. Uniquely, our present study will facilitate comparison of the effectiveness of a web-based PN advice across seven European countries, thus providing an insight into variations in the strategies required to surmount particular cultural barriers. Finally, the results of this study will inform the future development and implementation of web-based interventions aiming to improve lifestyle behaviours for the prevention of obesity and reduction in 
risk of NCDs such as diabetes, CVD and some common cancers.

Acknowledgments The Food4Me study is supported by the European Commission under the Food, Agriculture, Fisheries and Biotechnology Theme of the 7th Framework Programme for Research and Technological Development, Grant Number 265494.

\section{Conflict of interest None.}

Ethical standard All procedures performed in studies involving human participants were in accordance with the ethical standards of the institutional and/or national research committee and with the 1964 Helsinki declaration and its later amendments or comparable ethical standards.

\section{References}

Appel LJ, Brands MW, Daniels SR, Karanja N, Elmer PJ, Sacks FM (2006) Dietary approaches to prevent and treat hypertension-a scientific statement from the American Heart Association. Hypertension 47:296-308. doi:10.1161/01.hyp.0000202568. 01167.b6

Baecke JAH, Burema J, Frijters JER (1982) A short questionnaire for the measurement of habitual physical-activity in epidemiological-studies. Am J Clin Nutr 36:936-942

Bonn SE, Lagerros YT, Balter K (2013) How valid are web-based self-reports of weight? J Med Internet Res 15. doi:10.2196/jmir. 2393

Bonomi AG, Plasqui G, Goris AHC, Westerterp KR (2010) Estimation of free-living energy expenditure using a novel activity monitor designed to minimize obtrusiveness. Obesity 18:1845-1851. doi:10.1038/oby.2010.34

Dyson PA et al (2011) Diabetes UK evidence-based nutrition guidelines for the prevention and management of diabetes. Diabet Med 28:1282-1288. doi:10.1111/j.1464-5491.2011. 03371.x

Fallaize R, Macready AL, Butler LT, Ellis JA, Lovegrove JA (2013) An insight into the public acceptance of nutrigenomic-based personalised nutrition. Nutr Res Rev 26:39-48. doi:10.1017/ s0954422413000024

Fallaize R et al (2014) Online dietary intake estimation: reproducibility and validity of the Food4Me Food Frequency Questionnaire against a 4-day weighed food record. J Med Internet Res 16. doi:10.2196/jmir.3355

FAO (2004) Human energy requirements. Report of a joint FAO/ WHO/UNU expert consultation

Forster $\mathrm{H}$ et al (2014) Online dietary intake estimation: the Food4Me Food Frequency Questionnaire. J Med Internet Res 16:e150. doi:10.2196/jmir.3105

French SA, Jeffery RW, Wing RR (1994) Sex-differences among participants in a weight-control program. Addict Behav 19:147-158. doi:10.1016/0306-4603(94)90039-6

Gibney MJ, Walsh MC (2013) The future direction of personalised nutrition: my diet, my phenotype, my genes. Proc Nutr Soc 72:219-225. doi:10.1017/s0029665112003436

Hallal PC, Andersen LB, Bull FC, Guthold R, Haskell W, Ekelund U, Lancet Phys Activity Series W (2012) Global physical activity levels: surveillance progress, pitfalls, and prospects. Lancet 380:247-257. doi:10.1016/s0140-6736(12)60646-1
Hearty AP, McCarthy SN, Kearney JM, Gibney MJ (2007) Relationship between attitudes towards healthy eating and dietary behaviour, lifestyle and demographic factors in a representative sample of Irish adults. Appetite 48:1-11. doi:10.1016/j.appet. 2006.03.329

Kodama S et al (2012) Effect of web-based lifestyle modification on weight control: a meta-analysis. Int J Obes 36:675-685. doi:10. 1038/ijo.2011.121

Lassale C, Peneau S, Touvier M, Julia C, Galan P, Hercerg S, KesseGuyot E (2013) Validity of Web-based self-reported weight and height: results of the Nutrinet-Sante study. J Med Internet Res 15. doi: $10.2196 / \mathrm{jmir} .2575$

Marteau TM et al (2010) Effects of communicating DNA-based disease risk estimates on risk-reducing behaviours. Cochrane Database System Rev. doi:10.1002/14651858.CD007275.pub2

McCance RAW (2002) McCance and Widdowson's the composition of foods, 7th edn. Royal Society of Chemistry, London

Michie S, Ashford S, Sniehotta FF, Dombrowski SU, Bishop A, French DP (2011a) A refined taxonomy of behaviour change techniques to help people change their physical activity and healthy eating behaviours: the CALO-RE taxonomy. Psychol Health 26:1479-1498. doi:10.1080/08870446.2010.540664

Michie S, Hyder N, Walia A, West R (2011b) Development of a taxonomy of behaviour change techniques used in individual behavioural support for smoking cessation. Addict Behav 36:315-319. doi:10.1016/j.addbeh.2010.11.016

Nichols MTN, Luengo-Fernandez R, Leal J, Gray A, Scarborough P, Rayner M (2012) European cardiovascular disease statistics 2012. European Society of Cardiology, Brussels

Nielsen DE, El-Sohemy A (2012) A randomized trial of genetic information for personalized nutrition. Genes Nutr 7:559-566. doi:10.1007/s12263-012-0290-x

OECD (2012) Health at a glance: Europe 2012. OECD. doi:10.1787/ 9789264183896-en

Plasqui G, Westerterp KR (2007) Physical activity assessment with accelerometers: an evaluation against doubly labeled water. Obesity 15:2371-2379. doi:10.1038/oby.2007.281

Plasqui G, Joosen A, Kester AD, Goris AHC, Westerterp K (2005) Measuring free-living energy expenditure and physical activity with triaxial accelerometry. Obes Res 13:1363-1369. doi:10. 1038/oby.2005.165

Pursey K, Burrows TL, Stanwell P, Collins CE (2014) How accurate is web-based self-reported height, weight, and body mass index in young adults? J Med Internet Res 16. doi:10.2196/jmir.2909

Rosal MC, Ebbeling CB, Lofgren I, Ockene JK, Ockene IS, Hebert JR (2001) Facilitating dietary change: the patient-centered counseling model. J Am Diet Assoc 101:332-+. doi:10.1016/s00028223(01)00086-4

Rosenstock IM (1988) Adoption and maintenance of lifestyle modifications. Am J Prev Med 4:349-352

Sabinsky MS, Toft U, Raben A, Holm L (2007) Overweight men's motivations and perceived barriers towards weight loss. Eur J Clin Nutr 61:526-531. doi:10.1038/sj.ejcn.1602537

Senior V, Marteau TM, Peters TJ (1999) Will genetic testing for predisposition for-disease result in fatalism? A qualitative study of parents responses to neonatal screening for familial hypercholesterolaemia. Soc Sci Med 48:1857-1860. doi:10.1016/ s0277-9536(99)00099-4

Seybert HLA (2010) Internet usage in 2010-households and individuals. Eurosta

Stewart-Knox BJ et al (2009) Attitudes toward genetic testing and personalised nutrition in a representative sample of European consumers. $\mathrm{Br}$ J Nutr 101:982-989. doi:10.1017/ s0007114508055657 
Stewart-Knox B et al (2013) Factors influencing European consumer uptake of personalised nutrition. Results of a qualitative analysis. Appetite 66:67-74. doi:10.1016/j.appet.2013.03.001

Wei LJ, Lachin JM (1988) Properties of the urn randomization in clinical-trials. Control Clin Trials 9:345-364. doi:10.1016/01972456(88)90048-7

WHO (2008) 2008-2013 action plan for the global strategy for the prevention and control of noncommunicable diseases. WHO. Geneva, Switzerland
WHO (2009) Global health risks: mortality and burden of disease attributable to selected major risks. WHO. Geneva, Switzerland WHO (2010) Global recommendations on physical activity for health. World Health Organization. Geneva, Switzerland 